Leading article

\title{
The genetics of inflammatory bowel disease
}

The importance of genetic susceptibility in the pathogenesis of the chronic inflammatory bowel diseases Crohn's disease and ulcerative colitis - has recently been subject to detailed re-evaluation. Previously neglected in research on inflammatory bowel disease, the genetic basis of these diseases has now emerged as a focus of worldwide scientific activity. Epidemiological and clinical data from investigators in Europe and North America have provided strong evidence that Crohn's disease and ulcerative colitis are related polygenic disorders. Moreover, these findings have been complemented by the results of recent molecular genetic studies which have been designed and carried out in different populations with due regard to the potential confounding effects of ethnic differences, disease heterogeneity, and non-Mendelian inheritance. These studies have provided novel information regarding the number and identity of susceptibility genes involved in the pathogenesis of Crohn's disease and ulcerative colitis. The genetic basis of inflammatory bowel disease has rapidly become established as a major focus of research: given the current evidence that the identification of susceptibility genes in complex diseases is now practicable, these investigations offer great potential not only in understanding the pathogenesis of these diseases, but also in diagnosis and therapy.

Reported concordance rates in twin pairs have provided strong evidence that genetic predisposition is important in the pathogenesis of inflammatory bowel disease, particularly Crohn's disease. Most pertinent is the systematic analysis of the Swedish registry of twin births, reported by Tysk et al. ${ }^{1}$ These investigators identified 80 twin pairs in whom at least one twin was known to have inflammatory bowel disease. All twins were brought up in the same environment. Eight of 18 monozygotic twin pairs were concordant for Crohn's disease, but only one of 26 dizygotic pairs. In the twin pairs with one proband having ulcerative colitis, one of 16 monozygotic twins were concordant for disease but all other twenty pairs (dizygotic or unknown zygoticity) were discordant. From these data the authors derived a coefficient of heritability of Crohn's disease $(1 \cdot 0,95 \%$ confidence interval $(95 \% \mathrm{CI}) 0 \cdot 34-1 \cdot 0)$ and ulcerative colitis $0.53,95 \% \mathrm{CI} 0 \cdot 24-1 \cdot 0$ ) corrected for the common familial environment. These estimates of the genetic contribution to the pathogenesis of disease may be compared with twin studies in schizophrenia (coefficient of hereditability 0.68 ), insulin dependent diabetes $(0 \cdot 77)$, and hypertension $(0.57)$. Furthermore, the difference in concordance rates between Crohn's disease and ulcerative colitis provides evidence that these diseases may have a distinct genetic basis, with genetic factors being relatively more important in Crohn's disease. This study remains the most systematic twin study in inflammatory bowel disease; other reported studies ${ }^{2}{ }^{3}$ may have incurred ascertainment bias, and are subject to other methodological problems, some difficult to quantify from published data.

Many recent studies, involving different methodologies and populations have shown the increased prevalence of inflammatory bowel disease among relatives with Crohn's disease and ulcerative colitis. ${ }^{34}$ Consistent trends are noticeable in the published studies. First degree relatives are at greatest risk, particularly siblings, but more distant relatives also display an increased prevalence of disease. A positive family history is more common in patients with Crohn's disease than ulcerative colitis. Relatives of patients with Crohn's disease are at increased risk of developing either Crohn's disease or ulcerative colitis compared with the general population but are most likely to develop Crohn's disease.

The degree of familial clustering of disease may be expressed as the ratio of the risk of siblings of patients to the reported population prevalence, $\lambda$. In complex disorders, Risch has demonstrated that the magnitude of $\lambda$ is related to the degree of concordant inheritance for genetic determinants and therefore ease of genetic mapping. ${ }^{5}$ Two recent studies have estimated $\lambda$ in white populations resident in the United Kingdom. ${ }^{6}{ }^{7}$ In Oxford, the relative risks for Crohn's disease, ulcerative colitis, and inflammatory bowel disease overall in siblings of patients with Crohn's disease were estimated as respectively 36.5 , $16 \cdot 6$, and $24 \cdot 7$. These values may be compared with studies of other common disorders ${ }^{8}$ including type 1 diabetes $(\lambda=15)$, schizophrenia and type 2 diabetes (both $\lambda<10)$.

These epidemiological data do not suggest that either Crohn's disease or ulcerative colitis has a simple Mendelian pattern of inheritance and many authors regard the epidemiological data as providing conclusive evidence that simple models of disease are untenable. These results are most consistent with the model of Crohn's disease and ulcerative colitis as being related polygenetic disorders. The identity and number of susceptibility genes have been studied. Candidate gene based strategies have been applied, particularly to genes involved in regulation of the immune response. Data from detailed, well designed studies in North America, Japan, and Europe suggest that genes of the HLA system are important determinants of disease susceptibility and behaviour in ulcerative colitis, although less important in Crohn's disease. In Japanese ${ }^{9}$ and in Jewish patients, ${ }^{10}$ HLA DRB1 ${ }^{\star} 1502$ (DR2) is implicated as important in susceptibility to ulcerative colitis. In non-Jewish European patients, this allele is rare and not associated with ulcerative colitis. However, in these non-Jewish patients, ${ }^{11}{ }^{12}$ HLA genes seem to predict extent and severity of disease, and the presence of extraintestinal manifestations. Thus HLA typing in ulcerative colitis may have clinical applications in predicting disease behaviour. Prospective studies of newly diagnosed patients are currently under way, and will clarify this potential application of genotyping.

Systematic screening of the entire human genome has now emerged as a practicable strategy for the identification of susceptibility genes in complex polygenic disorders and behavioural traits. In 1992, Weissenbach et al were able to report the development of the first linkage map of the 
human genome, ${ }^{13}$ involving 814 highly polymorphic markers consisting of simple dinucleotide repeats. The markers described spanned $90 \%$ of the genome. The availability of this mapping strategy, together with the developments of semiautomated techniques for genotyping such markers, has allowed genome-wide searches in insulin dependent diabetes, multiple sclerosis and schizophrenia to be undertaken. As experience grows, the potential of this technique, together with the limitations, has become more fully appreciated. ${ }^{14} 15$ In particular, recent studies have emphasised the importance of access to large numbers of multiply affected families and rigorous statistical design and analysis.

Hugot et $\mathrm{al}^{16}$ reported the first genome-wide search for susceptibility genes in inflammatory bowel disease. Forty affected sibling pairs in 25 white families with Crohn's disease were studied using 270 autosomal markers. Analysis of allele sharing in affected sibling pairs identified only four markers achieving a $p$ value of 0.01 or less (three on chromosome 1 and one on chromosome 16). In a second set of families from the United Kingdom, supportive evidence for linkage with the region on chromosome 16 was obtained. This regio, designated IBD 1 by these authors, obtained a locus specific sibling $\lambda$ of $1 \cdot 3$. Putative candidate genes included in this region were the interleukin-4 receptor and CD11 integrin.

The study described by Hugot et $a l^{16}$ provided the first evidence that genome-wide screening could successfully be applied in inflammatory bowel disease. Moreover, the linkage described has now been replicated in other nonJewish European populations. However, the power of this investigation was limited by the number of families studied - the authors conceded that the locus identified on chromosome 16 was not likely to account for more than $10 \%$ of genetic susceptibility to Crohn's disease. The heterogeneity of patients studied and statistical thresholds employed are also areas of controversy. Finally, although the locus on chromosome 16 was designated IBD1, the contribution of this locus to ulcerative colitis was not assessed.

Satsangi et $a l^{17}$ have subsequently reported the results of a two stage genome-wide search for susceptibility genes involved in the pathogenesis of both Crohn's disease and ulcerative colitis. A total of 186 affected sibling pairs were studied: in 81 pairs, both siblings had Crohn's disease; in 64 , both siblings had ulcerative colitis; and in the remaining 41 sibling pairs, one sibling had Crohn's disease and the other ulcerative colitis.

In the first stage of investigation, 89 affected sibling pairs were genotyped at 260 microsatellite markers spanning the 22 autosomes. In the second stage, a further 97 affected sibling pairs were genotyped at 16 markers to investigate linkages suggested in the first data set. All families studied were white Europeans, resident in the United Kingdom.

The combined data for 186 affected sibling pairs provided striking evidence for linkage between inflammatory bowel disease and regions on chromosomes 12,7 , and 3. Furthermore, individual markers on chromosomes 2 and 6 were linked with susceptibility to ulcerative colitis but not Crohn's disease; and a region on chromosome 16 was linked with susceptibility to Crohn's disease, but not to ulcerative colitis.

These data have provided the most rigorous evidence to date that Crohn's disease and ulcerative colitis are related polygenic disorders, sharing some, but not all, susceptibility genes. The results also provide clear directions for further research. Replication of these linkages in independent data sets is an immediate priority for other investigators, and the regions on chromosomes 3, 7, and 12 are already subject to study in other populations. These chromosomal regions are relatively large, spanning respectively 6, 54, and 41 centiMorgans (cM). Before physical mapping or sequencing strategies are practicable, detailed studies are necessary to resolve the areas of linkage to within, ideally, $1 \mathrm{cM}$. Such precision mapping studies are dependent on the availability of even larger numbers of affected people and multiply affected families, together with considerable technical resources. ${ }^{18}$

Studies of candidate genes which have already been mapped to chromosomal locations within the linked regions may provide a short cut to susceptibility genes, bypassing the physical mapping of these regions. Strong positional candidates on chromosomes 7 and 3 are implicated by the recent studies. Of these, MUC3 (cytogenetic location 7q22) is one of two genes encoding the protein backbone of the intestinal secretory mucins. ${ }^{19}$ Selective deficiencies of mucin subclasses have been previously reported in both patients with ulcerative colitis and in their unaffected monozygotic twins. ${ }^{20}$ Other candidate genes within or close to the linked region on chromosome 7 included the genes encoding hepatocyte growth factor and the epidermal growth factor receptor.

The region on chromosome 3 contains the GNAI2 gene, which encodes a subunit of an inhibitory guanine nucleotide binding protein, known to be expressed in many tissues. Transgenic mice deficient in this protein have been described as having a lethal diffuse colitis, commonly complicated by adenocarcinoma of the colon. ${ }^{21}$

Research into the genetics of Crohn's disease and ulcerative colitis now holds the promise of providing real advances in our understanding of these disorders. The identification of susceptibility genes has emerged as a real possibility, and may have important clinical applications.

J SATSANGI D P JEWELL

Gastroenterology Unit, Radcliffe Infirmary, Oxford

J I BELL

Nuffield Department of Clinical Medicine,

fohn Radcliffe Hospital, Oxford

Correspondence to: Dr J Satsangi, The Gastroenterology Unit, Radcliffe Infirmary, Oxford OX2 6 HE.

1 Tysk C, Lindberg E, Jarnerot G, Floderus-Myrhed B. Ulcerative colitis and Crohn's disease in an unselected population of monozygotic and dizygotic twins. A study of heritability and the influence of smoking. Gut 1988;29: twins. A

2 Thompson NP, Driscoll R, Pounder RE, Wakefield AJ. Genetics versus environment in inflammatory bowel disease: results of a British twin study. BMF 1996; 312: 95-6.

3 McConnell RB, Vadheim CM. Inflammatory bowel disease. In: King RA Rotter JI, Motulsky AO, eds. The genetic basis of common diseases. Oxford: Oxford University Press, 1992: 326-48.

4 Satsangi J, Jewell DP, Rosenberg WMC, Bell JI. Genetics of inflammatory bowel disease. Gut 1994; 35: 696-700.

5 Risch N. Linkage strategies for genetically complex traits. II. The power of affected relative pairs. Am 7 Hum Gen 1990; 46: 229-41.

6 Probert CSJ, Jayanthi V, Pinder D, Wicks AC, Mayberry JI. Epidemiological study of ulcerative proctocolitis in Indian migrants and the indigenous study of ulcerative proctocolitis in Indian migrants

7 Satsangi J, Rosenberg WMC, Jewell DP. The prevalence of inflammatory bowel disease in relatives of patients with Crohn's disease. Eur $\mathscr{F}$ Gastroenterol Hepatol 1994; 6: 413-6.

8 Lander ES, Schork NJ. Genetic dissection of complex traits. Science 1994; 265: $2037-48$.

9 Futami S, Aoyama N, Honsako Y, et al. HLA-DRB1*1502 allele, subtype of DR15, is associated with susceptibility to ulcerative colitis and its progression. Dig Dis Sci 1995; 40: 814-8.

10 Toyoda H, Wang S-J, Yang H. et al. Distinct association of HLA class II genes with inflammatory bowel disease. Gastroenterology 1993; 104:

11 Satsangi J, Welsh KI, Bunce M, et al. Contribution of genes of the major histocompatibility complex to susceptibility and disease phenotype in inflammatory bowel disease. Lancet $1996 ; 347 ; 1212-7$.

12 Roussomoustakaki M, Satsangi J, Louis E, et al. Genetics of ulcerative colitis: HLA DRB1*0103 (DR103) is associated with extra-intestinal colitis: HLA DRB1*0103 (DR103) is associated with extra-int
manifestations and need for surgery. Gastroenterology (in press).

13 Weissenbach J, Gyapay G, Dib C, et al. A second generation linkage map of the human genome. Nature 1992; 359: 794-801.

14 Weeks DE, Lathrop GM. Polygenic disease: methods for mapping complex disease traits. Trends Genet 1995; 11: 513-9.

15 Lander ES, Kruglyak L. Genetic dissection of complex traits: guidelines for interpreting and reporting linkage results. Nat Genet 1995; 11: 241-7. 
16 Hugot JP, Laurent-Puig P, Gower-Rousseau C, et al. Mapping of a susceptibility locus for Crohn's disease on chromosome 16. Nature 1996; 379: 821-3.

17 Satsangi J, Parkes M, Louis E, et al. Two-stage genome-wide search in inflammatory bowel disease: evidence for susceptibility loci on chromosomes 3, 7 and 12. Nat Genet 1996; 14: 199-202.

18 Krugylak L, Lander ES. High resolution genetic mapping of complex traits. Am F Hum Genet 1995; 56: 1212-23.
19 Gendler SJ, Spicer AP. Epithelial mucin genes. Ann Rev Physiol 1995; 57: 607-34.

20 Tysk C, Riedesel H, Lindberg E, Panzini B, Podolsky D. Colonic glycoproteins in monozygotic twins with inflammatory bowel disease. Gastroenterology 1991; 100: 419-23.

21 Rudolph U, Finegold MJ, Rich SS, et al. Ulcerative colitis and adenocarcinoma of the colon in Goi2-deficient mice. Nat Genet 1995; 10: adenocar 143 